\title{
Axisymmetric Finite Element Modelling of Compound Magnetic Materials Based on Magnetic Micro-Wires by means of Equivalent homogeneous material
}

Alberto Delgado, Jesús A. Oliver, José A. Cobos, Jorge Rodríguez

\begin{abstract}
Compound magnetic materials based on parallel continuous ferromagnetic micro-wires embedded in a body made of polymeric material can potentially offer significant mechanical advantages over well-known fragile ferrite in some applications where the magnetic material has to withstand mechanical vibrations. Since the diameter of the magnetic micro-wires is very small (10's of $\mu \mathrm{m}$ ) compared to the rest of the dimensions (100's $\mathrm{mm}$ to 10 's $\mathrm{cm}$ ), and the number of micro-wires is in the order of 1000 's, brute force simulation by Finite Element Method is not possible. In this work, a method to overcome this limitation is proposed based on the use of a homogenous material that will behave, in a macroscopic sense, as the compound magnetic material. The physical properties of the homogeneous material are obtained in different directions, due to the inherent anisotropy of the compound magnetic component, by means of analytical equations.
\end{abstract}

Keywords-micro-wires; FEM; modelling; RFID systems; antennas; homogeneous models; characterization magnetic component;

\section{INTRODUCTION}

New compound magnetic materials using magnetic microwires can offer significant mechanical advantages over conventional ferrites while keeping good magnetic properties (see Figure 1). This advantage can be exploited to simplify, improve the reliability and reduce the cost of those applications in which the magnetic components are exposed to mechanical vibrations or impacts. Therefore, numerous investigations on flexible magnetic material, such as the micro-wires, are emerging [1] [2] [3].

In some applications, such as RFID systems (Radio Frequency IDentification) or WPT systems (Wireless Power Transfer), the magnetic core of the antenna is mainly excited in its longitudinal direction. Nevertheless, since the magnetic paths are not closed a significant part of the flux closes in the perpendicular direction. Due to the inherent anisotropic nature of the compound magnetic material (see Figure 2a), a matrix

This project (CDTI-DI-20160396) is funded by the Centro para el Desarrollo Tecnológico Industrial (CDTI). describing the modification of the physical properties in all the relevant directions is needed (see Figure $2 b$ ).

Hence, under the assumption that the homogeneous material is anisotropic, the different direction of the resulting magnetic field $\mathrm{H}$ is solved in the axisymmetric problem.

In this way, the model provides of a real relative permeability in longitudinal and radial direction, a real conductivity in the angular direction and a complex permeability in the longitudinal direction. The radial direction of the complex permeability is rejected.
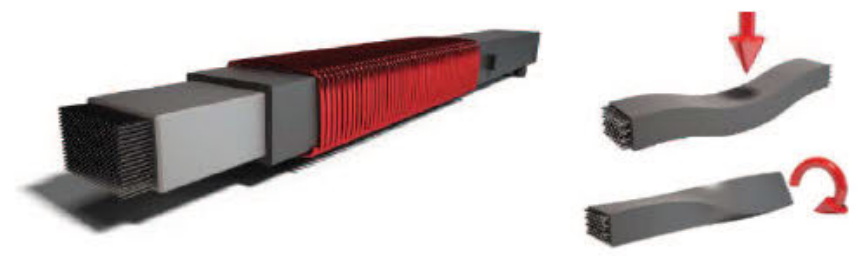

Fig. 1. Prototype designed by Group Premo of a Radio Frequency Dentification antenna based of micro-wires.
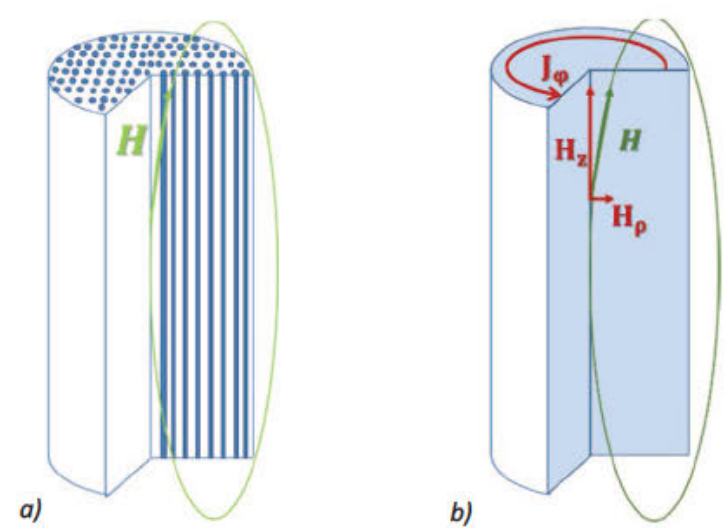

Fig. 2. a) Three-dimension view of a magnetic core made of micro-wires immersed in magnetic field $H$. b) Three-dimension view of a homogeneous magnetic core immersed in magnetic field $\mathrm{H}$. 


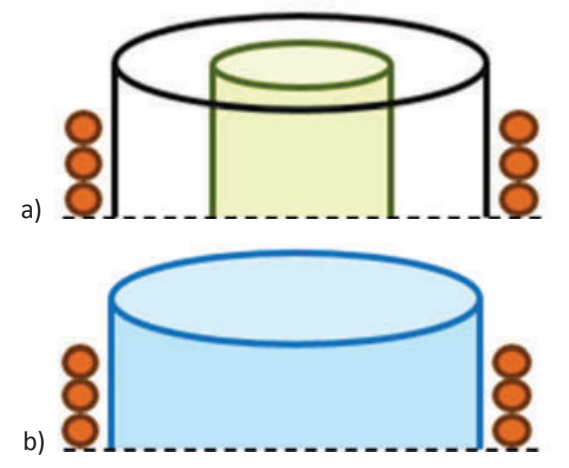

Figure 3. a) In green, the equivalent micro-wire made of $\mathrm{N}$ micro-wires. b) A homogeneous micro-wires made of a equivalent micro-wire and air.

\section{ANALYSIS OF THE PHYSICAL PROPERTIES IN THE LONGITUDINAL AND RADIAL DIRECTIONS}

\section{A. Modelling of micro-wires with magnetic field excitation in logitudinal direction}

As it is shown in Figure $2 \mathrm{~b}$ the magnetic field lines in a cylindrical solenoid (or antenna) are distributed both in the axial, $z$, and radial, $\rho$, directions. A model describing the equivalent physical properties of the magnetic micro-wires in both directions will be developed to allow performing Finite Element Simulations of these materials.

In this case, it is assumed that there exist a longitudinal magnetic field, $\mathrm{H}_{\mathrm{z}}$. Geometrically, the equivalent homogeneous material will keep the same geometrical dimensions as the composite material and the physical properties, permeability in the $\mathrm{z}$ direction and conductivity in the $\varphi$ direction will be modified in such a way that the losses and the energy will be kept the same.

For this purpose, the problem is divided into two parts: the first part is to group all micro-wires to do an equivalent microwire with the same area that the $\mathrm{N}_{\mu \mathrm{w}}$ micro-wires (see Figure $3 \mathrm{a}$ ), and the second part is to homogenize the equivalent microwire with the air to do a homogeneous micro-wire (see Figure $3 b)$.

The relative permeability of the polymeric material will be assumed equal to one and its conductivity equal to zero (there will not be induced currents on it).

The relative permeability, which has a real and complex parts, of the micro-wires is $\mu_{z \mu w}=\mu_{z \mu w}^{\prime}-j \mu_{z \mu w}^{\prime \prime}$, its conductivity is $\sigma_{\varphi \mu \mathrm{w}}$ and its radio is $r_{\mu \mathrm{w}}$.

The equivalent relative permeability of the equivalent microwire is $\mu_{\mathrm{zeq}}=\mu_{\mathrm{zeq}}^{\prime}-\mathrm{j} \mu_{\mathrm{zeq}}^{\prime \prime}$, its equivalent conductivity is $\sigma_{\varphi \mathrm{eq}}$ and its radio is

$$
r_{e q}=\sqrt{N_{\mu_{w}}} r_{\mu w}
$$

The homogeneous relative permeability of the homogeneous micro-wire is $\mu_{\mathrm{zh}}=\mu_{\mathrm{zh}}^{\prime}-\mathrm{j} \mu_{\mathrm{zh}}^{\prime \prime}$, its homogeneous conductivity is $\sigma_{\varphi \mathrm{h}}$ and its radio is $\mathrm{r}_{\mathrm{h}}$.

It is possible to obtain an analytical solution for the impedance of an infinite cylindrical magnetic core excited by a surface current density [4] [5]. The equivalent complex impedance of a core of length 1 and radius $r$, permeability $\mu$ and skin depth $\delta$ with 1 turn can be calculated using this analytical expression:

$$
Z=\frac{j \omega \mu r^{2} \pi}{l} F(\Delta)
$$

Where:

$$
F(\Delta)=\frac{2 I_{1}(\sqrt{2 j} \Delta)}{\sqrt{2 j} \Delta I_{0}(\sqrt{2 j} \Delta)}
$$

Where $I_{n}$ is the modified Bessel functions of the $n$ kind. $\Delta$ is a dimensionless parameter that is the ratio of the radius of the core to the skin depth.

The equation (3) can be expressed as:

$$
F(\Delta) \cong 1-\frac{j \Delta^{2}}{4}=F_{R}(\Delta)-F_{I}(\Delta)
$$

\section{1) Equivalent magnetic wire of $\mathrm{N}$ micro-wires}

The self-impedance of the $\mathrm{N}_{\mu \mathrm{w}}$ micro-wires is given by equation 2 and it has two components:

$$
\begin{aligned}
& Z_{z \mu w}^{I}=N_{\mu w} r_{\mu w}^{2}\left(\mu_{z \mu w}^{\prime} F_{R}\left(\Delta_{\mu w}\right)-\mu_{z \mu w}^{\prime \prime} F_{I}\left(\Delta_{\mu w}\right)\right) \\
& Z_{z \mu w}^{R}=N_{\mu w} r_{\mu w}^{2}\left(\mu_{z \mu w}^{\prime} F_{I}\left(\Delta_{\mu w}\right)+\mu_{z \mu w}^{\prime \prime} F_{R}\left(\Delta_{\mu w}\right)\right)
\end{aligned}
$$

The positive components represent the energy and the negative components represent the losses.

The self-impedance of the equivalent micro-wire is given by equation 2 and it has two components:

$$
\begin{gathered}
Z_{z e q}^{I}=r_{e q}^{2}\left(\mu_{z e q}^{\prime} F_{R}\left(\Delta_{e q}\right)-\mu_{z e q}^{\prime \prime} F_{I}\left(\Delta_{e q}\right)\right) \\
Z_{z e q}^{R}=r_{e q}^{2}\left(\mu_{z e q}^{\prime} F_{I}\left(\Delta_{e q}\right)+\mu_{z e q}^{\prime \prime} F_{R}\left(\Delta_{e q}\right)\right)
\end{gathered}
$$

The positive components represent the energy and the negative components represent the losses.

Since the energy and the losses must be kept the same, from equations 5 and 6 :

$$
\begin{array}{r}
N_{\mu w} r_{\mu w}^{2}\left(\mu_{z \mu w}^{\prime} F_{R}\left(\Delta_{\mu w}\right)-\mu_{z \mu w}^{\prime \prime} F_{I}\left(\Delta_{\mu w}\right)\right) \\
=r_{e q}^{2}\left(\mu_{z e q}^{\prime} F_{R}\left(\Delta_{e q}\right)-\mu_{z e q}^{\prime \prime} F_{I}\left(\Delta_{e q}\right)\right) \\
N_{\mu w} r_{\mu w}^{2}\left(\mu_{z \mu w}^{\prime} F_{I}\left(\Delta_{\mu w}\right)+\mu_{z \mu w}^{\prime \prime} F_{R}\left(\Delta_{\mu w}\right)\right) \\
=r_{e q}^{2}\left(\mu_{z e q}^{\prime} F_{I}\left(\Delta_{e q}\right)+\mu_{z e q}^{\prime \prime} F_{R}\left(\Delta_{e q}\right)\right)
\end{array}
$$

Assuming that the imaginary part of the permeability is smaller than the real part and $\Delta<1$, the term $\mu^{\prime \prime} F_{I}(\Delta)$ can be neglected with respect to $\mu^{\prime} \mathrm{F}_{\mathrm{R}}(\Delta)$ in equation (7) and then, using (1):

$$
\mu_{z e q}^{\prime}=\mu_{z \mu w}^{\prime}
$$

From equation 8, it is possible to separate the Eddy Current losses and the hysteresis losses and residual losses. Thus:

$$
\begin{gathered}
\sigma_{\varphi e q}=\sigma_{\varphi \mu w} / N_{\mu w} \\
\mu_{z e q}^{\prime \prime}=\mu_{z \mu w}^{\prime \prime}
\end{gathered}
$$


2) Equivalent homogeneous material from equivalent wire

The self-impedance of the homogeneous micro-wire is given by equation 2 and it has two components:

$$
\begin{aligned}
& Z_{z h}^{I}=r_{h}^{2}\left(\mu_{z h}^{\prime} F_{R}\left(\Delta_{h}\right)-\mu_{z h}^{\prime \prime} F_{I}\left(\Delta_{h}\right)\right) \\
& Z_{z h}^{R}=r_{h}^{2}\left(\mu_{z h}^{\prime} F_{I}\left(\Delta_{h}\right)+\mu_{z h}^{\prime \prime} F_{R}\left(\Delta_{h}\right)\right)
\end{aligned}
$$

The positive components represent the energy and the negative components represent the losses.

The self-impedance of the polymeric material part is given by equation 2 and it has only one component:

$$
Z_{z p m}=j\left(r_{h}^{2}-r_{e q}^{2}\right) F_{R}\left(\Delta_{p m}\right)
$$

This component represents the energy in the air.

Since the energy and the losses must be kept the same, from equations 6,12 and 13 :

$$
\begin{aligned}
& r_{h}^{2}\left(\mu_{z h}^{\prime} F_{R}\left(\Delta_{h}\right)-\mu_{z h}^{\prime \prime} F_{I}\left(\Delta_{h}\right)\right)= \\
& =r_{e q}^{2}\left(\mu_{z e q}^{\prime} F_{R}\left(\Delta_{e q}\right)-\mu_{z e q}^{\prime \prime} F_{I}\left(\Delta_{e q}\right)\right)+\left(r_{h}^{2}-r_{e q}^{2}\right) F_{R}\left(\Delta_{p m}\right) \\
& r_{h}^{2}\left(\mu_{z h}^{\prime} F_{I}\left(\Delta_{h}\right)+\mu_{z h}^{\prime \prime} F_{R}\left(\Delta_{h}\right)\right)= \\
& =r_{e q}^{2}\left(\mu_{z e q}^{\prime} F_{I}\left(\Delta_{e q}\right)+\mu_{z e q}^{\prime \prime} F_{R}\left(\Delta_{e q}\right)\right)
\end{aligned}
$$

Since $\mu^{\prime \prime} F_{I}(\Delta) \ll \mu^{\prime} F_{R}(\Delta)$, from the equation 14 :

$$
\mu_{z h}^{\prime}=\mu_{z e q}^{\prime}\left(\frac{r_{e q}}{r_{h}}\right)^{2}+\left(1-\left(\frac{r_{e q}}{r_{h}}\right)^{2}\right)
$$

From equation 15, it is possible to separate the Eddy Current losses and the hysteresis losses and residual losses. Thus:

$$
\begin{gathered}
\sigma_{\varphi h}=\sigma_{\varphi e q}\left(\frac{\mu_{z e q}^{\prime}}{\mu_{z h}^{\prime}}\right)^{2}\left(\frac{r_{e q}}{r_{h}}\right)^{4} \\
\mu_{z h}^{\prime \prime}=\mu_{z e q}^{\prime \prime}\left(\frac{r_{e q}}{r_{h}}\right)^{2}
\end{gathered}
$$

\section{B. Modelling of micro-wires with magnetic field excitation in perpendicular direction}

In the case that the flux needs to travel in the radial direction (see figure $4 a$ ), it will find micro-wires of high permeability separated by a gap of polymer of relative permeability equal to one. Therefore, the resultant permeability in this direction will be very low.

Here, two solutions of the investigated problem are presented and compared: the first is a model where the permeability is computed using the capacitance analogy [6] [7] by a sets of simulations with Ansys Maxwell ${ }^{\circledR}$, the second is a model based on the theory of the effective magnetic permeability of multiphase materials solved by Z. Hashin and S. Shtrikman [8] [9].

The relative permeability of the micro-wires is $\mu_{\rho \mu w}^{\prime}$ and the relative permeability of the homogeneous micro-wire is $\mu_{\rho h}^{\prime}$.

In both methods, it assumes that the radial complex permeability in the frequency range studied is very small, and then it is rejected.

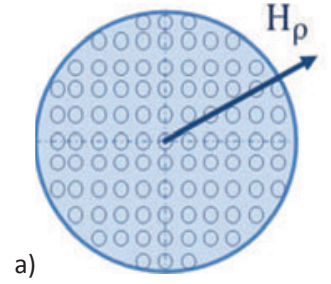

b)

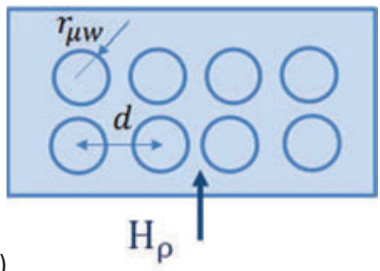

Figure 4. a) Cross section of compound material. b) Detailed cross section.

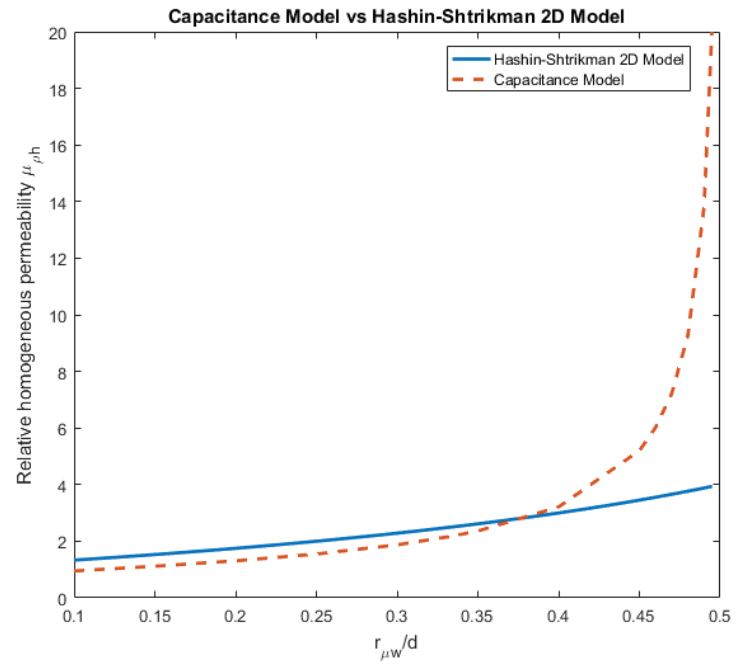

Figure 5. Relative homogeneous permeability as a function of the $\frac{r_{\mu w}}{d}$ in discontinuous line. Relative homogeneous permeability from HashinShtrikman in continuous line.

1) Reluctance calculation using the capacitance analogy

Since the permeability of the micro-wires is in the order of 1000 's times higher than the permeability of the polymer, it will be assumed that the reluctance of this path can be calculated as the capacitance between the micro-wires.

To estimate the variation of the reluctance with the radius of the microwires, $r_{\mu w}$, and their distance, $d$, (see figure 5) a set of electrostatic test in MAXWELL ANSYS ${ }^{\circledR}$ where performed. Based on these tests the equivalent permeability was obtained as a function of the dimensionless parameter $\frac{r_{\mu w}}{d}$ and the results are shown in Figure 5.

2) Theory of the effective magnetic permeability of multiphase materials

This method assumes that the composite of isolated cylinders which have similar radius and the coating material are similar, the relative homogeneous permeability is given by:

$$
\mu_{\rho h}^{\prime}=1+\frac{2 f_{v \mu w}\left(\mu_{\rho \mu w}^{\prime}-1\right)}{2+\left(1-f_{v \mu w}\right)\left(\mu_{\rho \mu w}^{\prime}-1\right)}
$$

Where $f_{v \mu w}$ is the volume fraction of the micro-wire. The results are shown in Figure 5. 


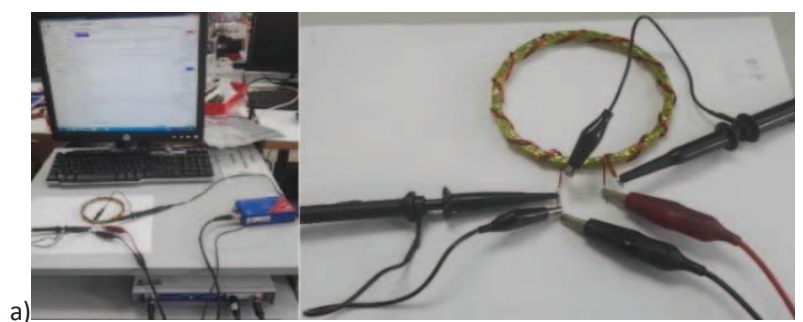

b)

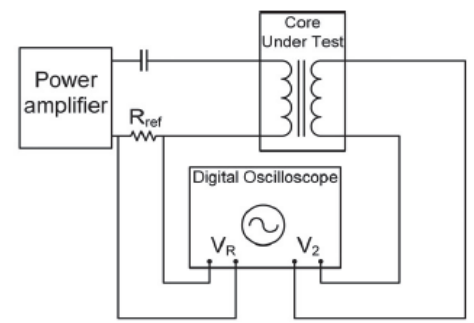

Figure 6. a) The characterization setup with a Bode100 of Omicron $®$. b) The schematic of the characterization setup.
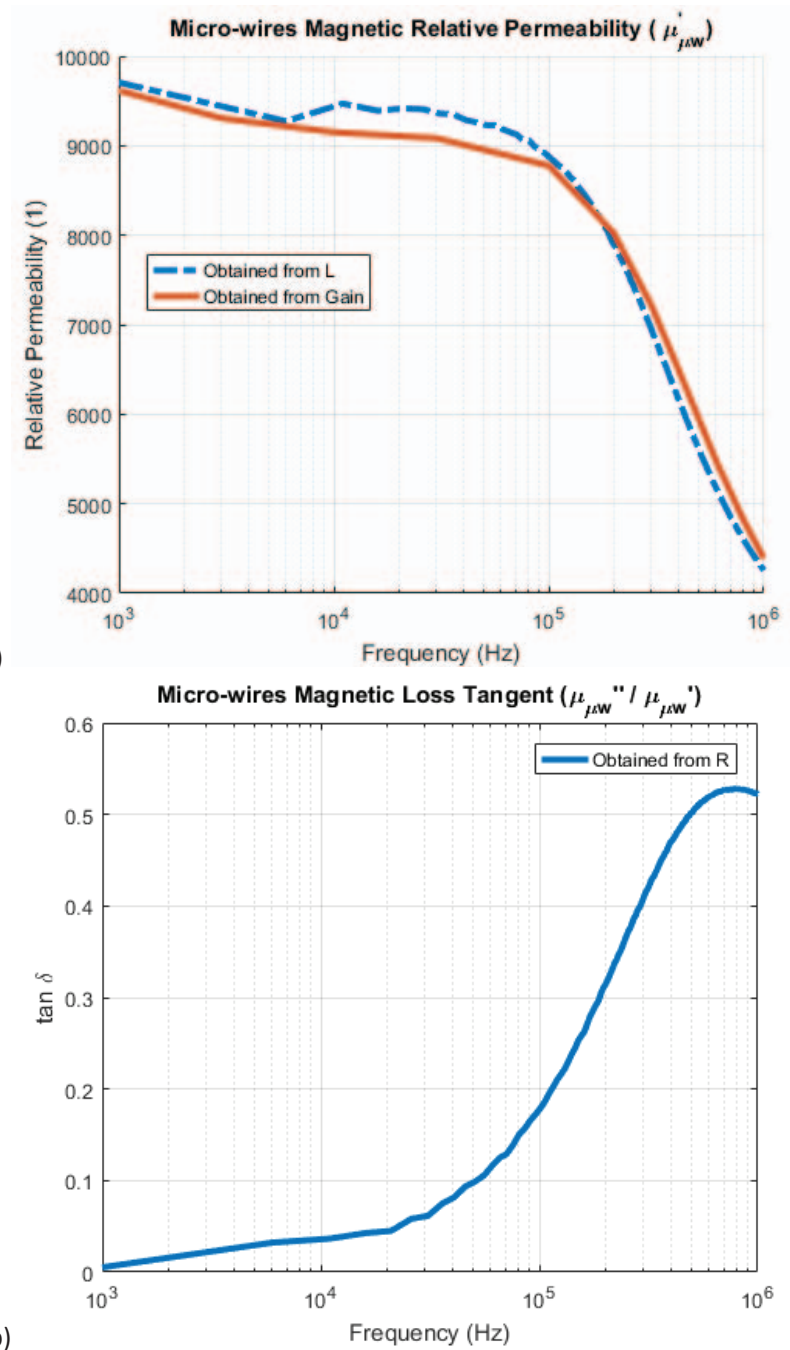$$
\text { b) }
$$

Figure 7. a) The relative permeability of the micro-wires. b) The loss tangent of the micro-wires.

\section{MICRO-WIRES CHARACTERIZATION AND PARAMETER ESTIMATION}

The properties of the magnetic micro-wires have been extracted using a Vector Network Analyzer (Bode100 from Omicron $\left.{ }^{\circledR}\right)$. The micro-wires are made of an alloy of amorphous material based on CoFeBSiNiMn. The micro-wires have an isotropic behavior. The characterization has been performed using the setup shown in Figure 6a. The voltage in the secondary and the input current in the primary are measured according to $[10]$ as shown in the Figure $6 b$.

The relative permeability of the microwires can be shown to be given by:

$$
\mu_{\mu w}^{\prime}=\frac{V_{2}}{i_{1}} \frac{l_{c}}{N_{2} N_{1} \mu_{0} \omega} \frac{1}{A_{\mu w}}-\frac{A_{\text {air }}}{A_{\mu w}}
$$

The conductivity of the micro-wires has been obtained by measuring the $\mathrm{DC}$ resistance of one meter of the micro-wire. The value obtained is $694500 \mathrm{~S} / \mathrm{m}$. The relative permeability is represented in Figure 7a. The loss tangent is represented in Figure $7 b$.

\section{Finite Element Modelling Results}

Since the relative permeability, complex permeability and conductivity are known, a toroid simulation in Ansys Maxwell (B) using this characterize is done. The dimensions of this toroid are: $\mathrm{ID}=90 \mathrm{~mm}, \mathrm{OD}=95 \mathrm{~mm}, \mathrm{~h}=5 \mathrm{~mm}$ and the 13 -turn winding is made with round copper wire. The core is made of 310 micro-wires. The measured result and the simulated result are compared in the figure 8 .

Additionally, an RFID antenna has been built for comparison. The dimensions are: $120 \mathrm{~mm}$ x $9 \mathrm{~mm}$ x $5 \mathrm{~mm}$. The magnetic core is made of 400 micro-wires. The 70-turn winding is made of copper wire with a diameter of $0.45 \mathrm{~mm}$. The measured and simulated result are shown in the Figure 9.

\section{CONCLUSIONS}

In this work, a method to allow finite element simulation of compound materials with magnetic micro-wires is proposed. The method is based on the use of a homogenous material with anisotropic properties that will keep macroscopic energy and losses of the compound material in axial and radial directions. The physical properties of the homogeneous material are obtained by means of analytical equations.

In the case of the toroid, the results are very accurate, with a precision of $99 \%$ in the inductance and $96 \%$ in the resistance. In the case of the antenna, the results are accurate, with an offset of $+10 \mu \mathrm{H}$ in the inductance and $98 \%$ in the resistance. 


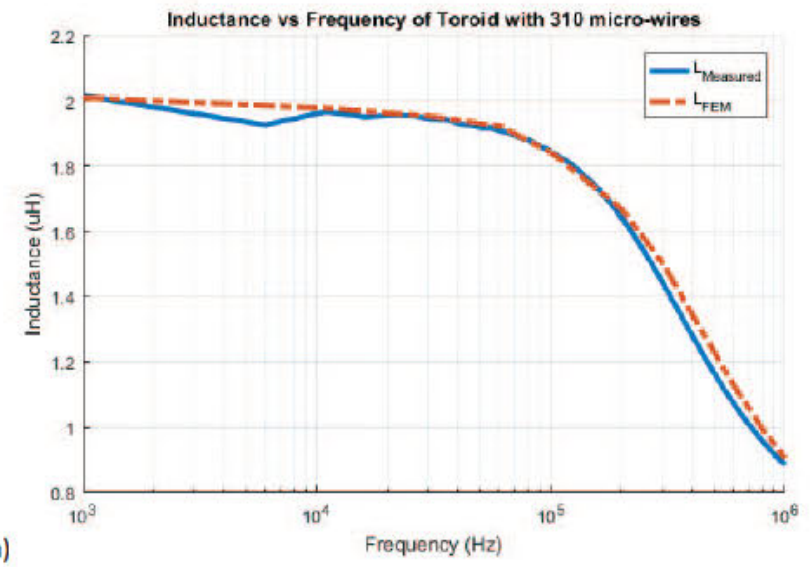

a)

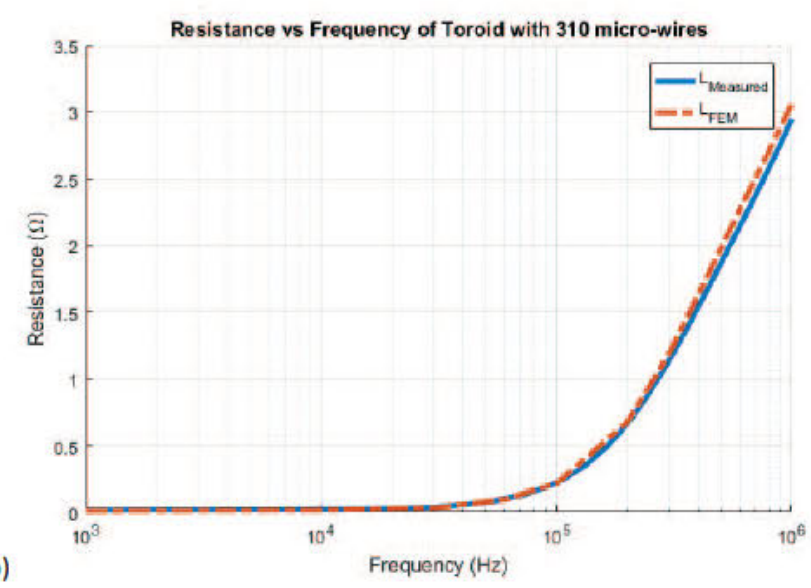

Figure 8. a) Inductance of Toroid with 310 micro-wires. b) Resistance of Toroid with 310 micro-wires

\section{REFERENCES}

[1] Infante, G. (2010). Propiedades magnéticas y de transporte de nuevos microhilos mono y bifásicos.

[2] He, D., \& Shiwa, M. (2014). A Magnetic Sensor with Amorphous Wire. Sensors, 14(6), 10644-10649.

[3] Devkota, J., Luong, T., Liu, J. S., Shen, H., Qin, F. X., Sun, J. F., ... \& Phan, M. H. (2014). A soft ferromagnetic multiwire-based inductance coil sensor for sensing applications. Journal of Applied Physics, 116(23), 234504.

[4] Hurley, W. G., \& Wölfle, W. H. (2013). Transformers and inductors for power electronics: theory, design and applications. John Wiley \& Sons.

[5] Wu, J., \& Lei, Y. (2002). Analytical expression to impedance for solenoid coil with a coaxial cylindrical ferrite core of finite length. Journal of Physics D: Applied Physics, 35(6), 570.

[6] Muhlethaler, J., Kolar, J. W., \& Ecklebe, A. (2011, May). A novel approach for 3D air gap reluctance calculations. In Power Electronics and ECCE Asia (ICPE \& ECCE), 2011 IEEE 8th International Conference on (pp. 446-452). IEEE

[7] Balakrishnan, A., Joines, W. T., \& Wilson, T. G. (1997). Air-gap reluctance and inductance calculations for magnetic circuits using a Schwarz-Christoffel transformation. IEEE Transactions on Power Electronics, 12(4), 654-663.

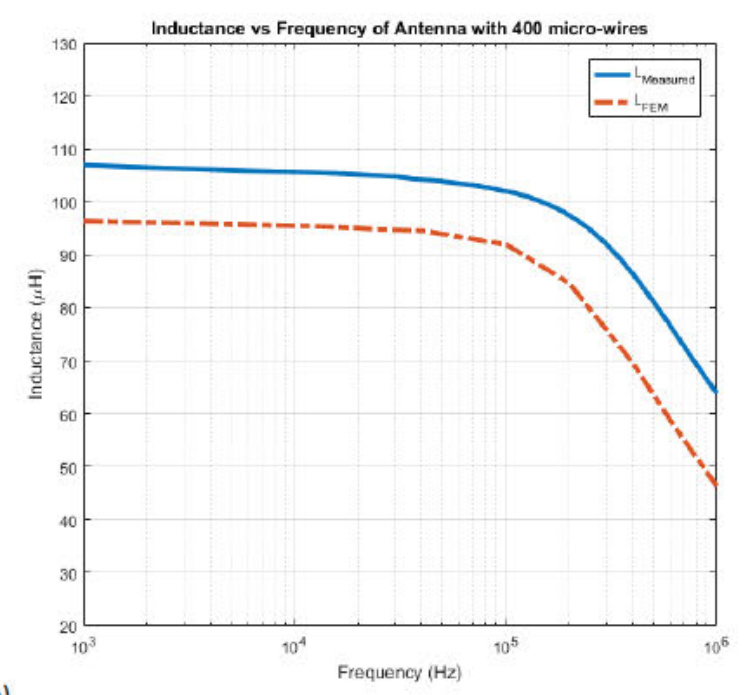

a)

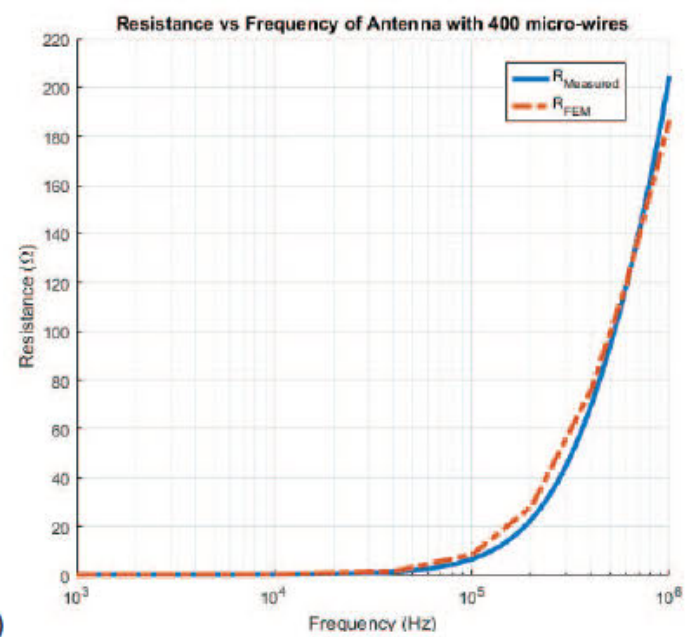

Figure 9. a) Inductance of Antenna with 400 micro-wires. b) Resistance of Antenna with 400 micro-wires

[8] Hashin, Z., \& Shtrikman, S. (1963). A variational approach to the theory of the elastic behaviour of multiphase materials. Journal of the Mechanics and Physics of Solids, 11(2), 127-140.

[9] Fiske, T. J., Gokturk, H. S., \& Kalyon, D. M. (1997). Percolation in magnetic composites. Journal of materials science, 32(20), 5551-5560.

[10] Mu, M., Li, Q., Gilham, D. J., Lee, F. C., \& Ngo, K. D. (2014). New core loss measurement method for high-frequency magnetic materials. IEEE Transactions on Power Electronics, 29(8), 4374-438 